\title{
Roby-Go, a Prototype for Several MiroSOT Soccer Playing Robots
}

\author{
Gregor Novak \\ Vienna University of Technology \\ Institute of Computer Technology \\ Vienna, Austria \\ novak@bluetechnix.at
}

\begin{abstract}
For a competitive robot soccer team a perfect working robot is a fundamental part. The developed mobile mini robot is a two wheeled differentially driven (2WDD) robot, distinguished by its simple, compact and modular construction. Due to the open architecture of the robot it is possible to use it not only as a soccer player, in the category MiroSOT, but also as a mobile platform in a number of several other tasks, for example as an independent testing bed for other Multi Agent Systems.
\end{abstract}

\section{INTRODUCTION}

In 1994 robot soccer was introduced with the theoretical background to develop multi-robot adaptive, co-operative, autonomous systems solving common tasks. A group of robots must interact and self-organise in order to achieve the common given goal. The other technical aspects beside of co-operative and co-ordinated behaviour are miniaturization of robot, precise movement and increasing power efficiency. There are several categories in robot soccer: NaroSOT, MiroSOT, KheperaSOT and HuroSOT, classified by the size of the robots and number of playing robots. In category of MiroSOT the size of a robot is limited to $7.5 \mathrm{~cm}$ cubic ${ }^{1}$. The playground is supervised by a camera mounted above the playground. The image of the camera is processed by a host computer located beside the playground. The result of the program on the host computer is a trajectory, which the robot has to follow as accurate as possible [1].

However for a robot soccer program it makes no difference if there is a real mini robot or a simulator. But indeed for a competition in robot soccer a perfect working robot is a fundamental part of the whole system. The mini robot playing soccer has to fulfill its task as accurate as possible without any controlling help from the control loop at the host computer. This results out of three main facts. One fact is that a sampling time of even $20 \mathrm{~ms}$ is much too less to control a robot driven by DC motors. The second fact is that the dead time between shooting a picture and reacting of the robot is much too long and last, the inaccuracy of the vision system is much too big. This leads to the fact that a robot must have an own controller for its DC motors on board.

\footnotetext{
${ }^{1}$ Information about the FIRA rules can be found at http://www.fira.net
}

In summer 1999 the design of this soccer robot, called Roby-Go started. The first version was finished in spring 2000. In 2001 it was equipped with additional acceleration sensors in order to prevent slippering of the wheels [2]. In this paper the first version of Roby-Go is described. This robot should become the prototype for nearly all European soccer robots in the category of MiroSOT [3].

\section{THE REQUIREMENTS FOR ROBY-GO}

Out of the experience of the European championship 1999 in Dortmund (Germany) and the world championship 1999 in Campinas (Brazil) a competitive soccer robot has to meet the following requirements:

- Fulfill its task as accurate as possible

- Stay on its given trajectory

- Control itself

- Possibility to measure its movement is needed

- Fit into a cube with an edge length of $7.5 \mathrm{~cm}$

- Have a low-lying center of gravity

- Have a separate power electronic from controlling electronic

- Have no front or back side (symmetric robot)

- Have a possibility for driving the ball

- Have broad wheels and tires with high friction

- Have roller bearings for mounting the wheels

- Have a singe stage gear

- Motor with high resolution encoders

- Have a modular and open architecture for upgrading the robot

- Reach a speed up to $2.5 \mathrm{~m} / \mathrm{s}$

- Have an acceleration of more than $5 \mathrm{~m} / \mathrm{s}^{2}$

- Have a tolerance of motion less than $\pm 0.5 \mathrm{~cm} / \mathrm{m}$ 
A robot soccer playing mini robot of the category MiroSOT consists of the following parts

- Power supply

- Two DC motors with digital encoder

- Single stage gear

- Two wheels

- Micro controller for controlling the rotation of the DC motors

- Power electronic

- Radio module for communication (send tasks to the robot)

By all these facts the sight to develop a robot, which cannot only be used for robot soccer, but also as a mobile platform for multi agents in general, was never lost. Furthermore the possibility of adding sensors very easily to the robot follows from the modular architecture.

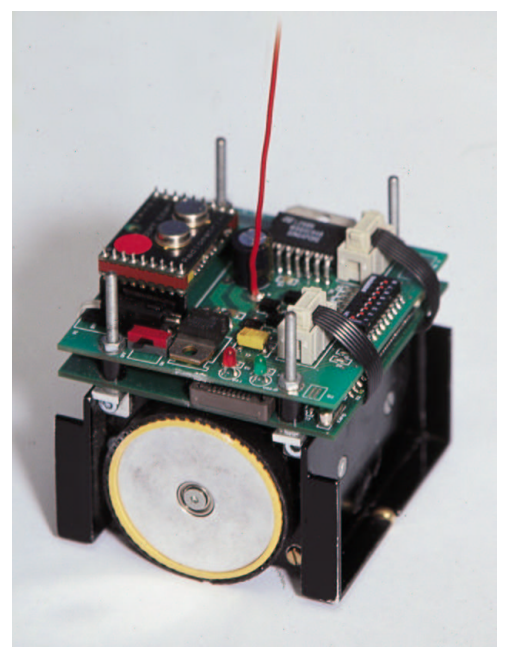

Fig. 1: The Mini Robot

According to the experiences mentioned above and the FIRA rules [3], a new mini robot (Fig. 1) was developed. This mini robot is a two wheeled differentially driven (2WDD), distinguished by his simple, compact and modular architecture. It has two PWM controlled DC motors.

\section{OVERALL STRUCTURE OF THE MINI ROBOT}

Concerning the FIRA (Federation of International Robotsoccer Association) MiroSOT rules, the whole robot has to fit with all its components into a cube of an edge length of $7.5 \mathrm{~cm}$. Therefore results a height of the mechanical part of less than $5 \mathrm{~cm}$. Furthermore a division of the robot's electronic into two parts, the motion unit and its intelligence (sensor, etc.) seems appropriate. In the first step this 'intelligence' consists of a radio module and its decoding part only. A DIP switch is used to assign the robot a unique identifier. The motion unit itself is connected by some kind of bus with the 'remaining part' of the robot. For controlling the motion unit velocity, angular velocity and commands (Go, Stop, Reset) will be provided. The motion unit is done as compact as possible in order to safe room for further electronic.

The robot is seen as a smart actor. It represents a complete control loop getting its demand value from a layer above. This layer above is in this step of development implemented on the control program at the host computer, but the idea is to move layer by layer to the mobile robot.

\section{MECHANICS}

The mechanical part of the mobile platform consists of the following parts:

- Two wheels with special tire

- Two one stage gears

- Two DC motors with two channel magnetically encoders with a resolution of 512 impulses per rotation

- Two side panels

- Two front panels

- Battery and battery mounting

- Special mounting for the electronic

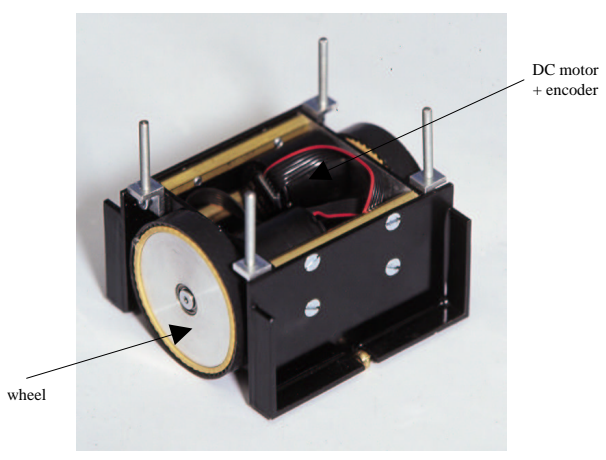

Fig. 2: Mechanics

According to the free space of the mechanic (Fig. 2) the motor has to have a maximum length of $40 \mathrm{~mm}$ and a maximum diameter of $23 \mathrm{~mm}$. The current has to be between 3 and $12 \mathrm{~V}$ and the rotation speed should be between 6000 and $10000 \mathrm{rot} / \mathrm{min}$. 
The starting torque of the motor is estimated with the following assumptions. A soccer robot needs an acceleration of about $5 \mathrm{~m} / \mathrm{s}^{2}$. Furthermore the one stage gear has a gear ratio of about 1:10 (pinion:wheel) and the weight of the robot is about $500 \mathrm{~g}$. The motor torque is:

$$
M_{M}=\frac{a \cdot m \cdot r_{w h e e l} \cdot i}{2}
$$

$$
\begin{aligned}
& M_{M} \quad \text { Motor torque } \\
& \mathrm{m} \quad \text { Mass of the robot } \sim 500 \mathrm{~g} \\
& r_{\text {wheel }} \text { Diameter of the wheel } \sim 50 \mathrm{~mm} \\
& \text { a Acceleration } \sim 5 m / s^{2} \\
& \text { i } \quad \text { Gear ratio } \sim 1: 10 \\
& M_{M}=\frac{5 \cdot 0.5 \cdot 0.05 \cdot 0.1}{2}=6.25 \cdot 10^{-3} \mathrm{Nm}
\end{aligned}
$$

The minimum number of impulses per rotation of encoder is estimated by means of the following assumption. The maximum velocity of the robot should be $2.54 \mathrm{~m} / \mathrm{s}$. Furthermore the one stage gear has a gear ratio of about 1:10 (pinion:wheel) and the radius of a robot wheel is $50 \mathrm{~mm}$. The rotation speed $n$ of the motor is estimated:

$$
\begin{aligned}
& n=\frac{v_{\text {wheel }}}{2 \cdot \pi \cdot \cdot i \cdot r_{\text {wheel }}} \\
& n=\frac{2.54}{2 \cdot \pi \cdot 0.1 \cdot 0.025}=162 \mathrm{rot} / \mathrm{sec} \\
& n \quad \text { rotation per second } \\
& v_{\text {wheel }} \quad \text { velocity of the wheel } 2.54 \mathrm{~m} / \mathrm{s}
\end{aligned}
$$

The minimum number of impulses per millisecond is based on the supposed sampling time of one millisecond and the resolution of the demand value. If a minimum number of impulses per millisecond $N_{\min }$ of 254 is required, the number of impulses $N$ for the encoder per rotation is calculated by

$$
\begin{aligned}
& N=\frac{N_{\min }}{n} \\
& N=\frac{254}{0.162}=1568 \mathrm{Imp} / \mathrm{rot}
\end{aligned}
$$

\section{ELECTRONIC}

A soccer robot in MiroSOT is basically not more than a mobile platform, which is connected via a radio module to its superior control unit, which is located at the host PC. The electronic is built up in an open architecture. In theory a robot consists of different units fulfilling special tasks. One important unit is the motion unit. The motion unit controls the motors by a desired trajectory. This desired trajectory as well as other demand behaviors like acceleration etc. have to be transferred to the motion unit. Transferring data to the motion unit or any other unit is in principle done via a bus system. As bus system the CAN bus can be used. The CAN bus is very common in the automotive industry, but of course any other bus system, which fulfills the soft real time criteria in a certain way, is possible. Also a hierarchical bus system with different busses for different tasks is thinkable. This means that such a mobile platform fits into a layer model and the motion unit is part of a networked control system (Fig. 3).

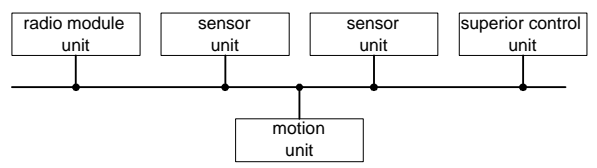

Fig. 3: Networked Control System

For the design of a soccer robot the considerations above mean that it consists of a motion unit and a connection via radio to its superior control unit. Remaining to the described system, the radio module should be connected to a micro controller, which selects and processes the incoming information. Afterwards a bus provides the processed information to the motion unit (Fig. 4).

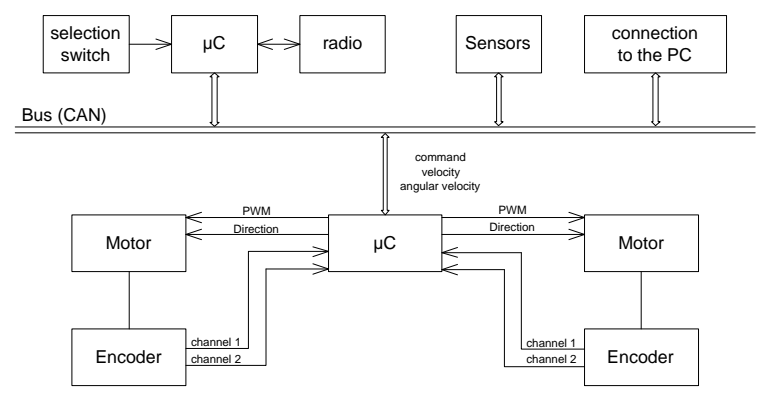

Fig. 4: Bus System for a Mobile Platform

As shown in Figure 4 a modular design was realized, beside the fact that the bus between the radio connection and the micro controller was economized. However, the electronic was separated on two boards, one board for the micro controller and a second board containing the power electronic, additional sensors, and the radio module. On the one hand, the separation in two boards is based on economical factors. 
The micro controller board is a four-layer print and the other board is only a two-layer print. On the other hand, the development for sensors etc. is limited to the other board and the micro controller board can remain the same. Furthermore the micro controller board can be used for further applications.

The micro controller board represents a complete starter kit, which is in circuit programmable and can be used for a several number of other applications. It contains a micro controller, a flash EPROM, a serial connection interface for programming and a CAN bus interface. The used micro controller is a C167 of Infineon. This micro controller has in silicon 6 PWM (Pulse Width Modulated) outputs, two inputs for digital encoders, AD (analog digital) and DA (digital analog) converter, and a lot of free input and output pins, which partly generate interrupts on rising or falling edges. Furthermore it contains in silicon a CAN bus interface, an asynchronous and a synchronous serial interface. The primary task of the micro controller is to control the movement of the robot. Therefore four PWM output signals, two direction signals and 4 pins for the two encoders are required. The micro controller is clocked with $20 \mathrm{MHz}$. To run this board a voltage supply of $5 \mathrm{~V}$ is required.

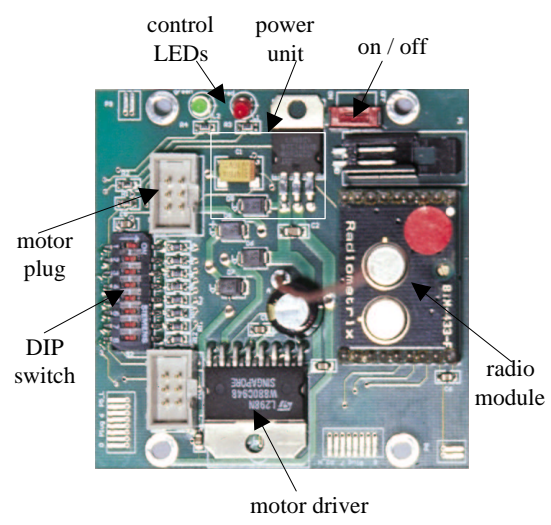

Fig. 5: Motor Board

The second board (Fig. 5) uses the I/O pins of the micro controller board and contains the implementation. The DC motors are controlled by PWM signals, generated by the micro controller. Due to the fact that the motors need much more electric current than the micro controller could provide, a driver is required and a dual full bridge driver is installed. For the radio communication a special module is used, which can be plugged in this board. For selecting a robot, remember the robots are playing in a team, 8 DIP-switches are integrated. Additionally two LEDs signal if the robot is turned on and if the radio communication works properly. The robot is supplied with $10.8 \mathrm{~V}$. A potential transformer transforms this voltage to the $5 \mathrm{~V}$ that the micro controller requires.

\section{THE MOTION CONTROLLER OF THE MINI} ROBOT

In order to win a robot soccer game it is important to have a soccer robot, which reacts very fast on new demand values and stays on its trajectory as accurate as possible. The mobile platform gets two demand values describing a trajectory from its superior control loop. The robot's behavior is raised to the two DC motors. Each motor has its encoder, which counts the rotations of the motor. Due to this feedback the motors are controlled. The manipulated variables are the voltages for the motors. The different voltages that are necessary to control the motor are equivalent to a PWM signal. A PWM signal is a direct current with constant voltages, which is pulsed. The integral over the pulsed direct current signal with constant voltage is equivalent to a direct current signal with a specific current. The controller of this mobile platform, which is designed to react very fast on new demand values and to stay on its trajectory as accurate as possible, is implemented in the robot's micro controller. The sampling time for the controller is primary based on the encoder resolution and the resolution of the demand value.

The demand values for the mobile platform are the translatoric velocity $v_{R}$ and the angular velocity $\omega_{R}$. These both describe the trajectory for the robot. The interval of the translatoric velocity is between $-2.54 \mathrm{~m} / \mathrm{s}$ and $+2.54 \mathrm{~m} / \mathrm{s}$. The accuracy of this demand value is $0.01 \mathrm{~m} / \mathrm{s}$. For the angular velocity applies that the interval is between $-25.4 \mathrm{rad} / \mathrm{s}$ and $+25.4 \mathrm{rad} / \mathrm{s}$ with an accuracy of $0.1 \mathrm{rad} / \mathrm{s}$. A new demand value is set every $20 \mathrm{~ms}$. The calculated maximum velocity of a robot is $2.41 \mathrm{~m} / \mathrm{s}$. This value is calculated for the $6 \mathrm{~V}$ motor used with $6 \mathrm{~V}$ [4]. For the mini robot the $6 \mathrm{~V}$ motor is taken, but used with $10.8 \mathrm{~V}(=9 \mathrm{x} 1.2 \mathrm{~V})$. Using a DC motor like this with the double of the designed voltage means a bisection of the motor's lifetime.

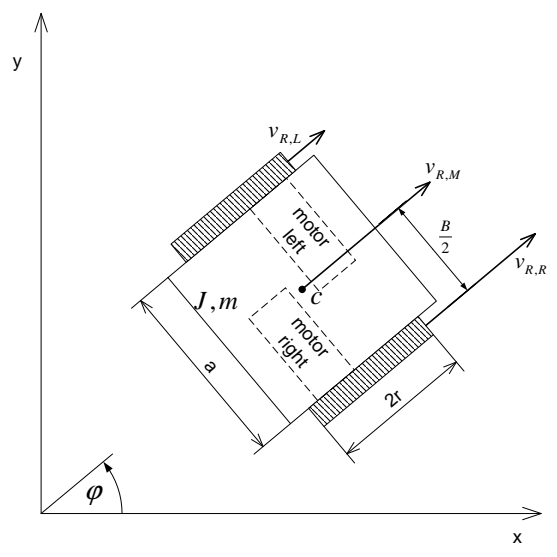

Fig. 6: Mini Robot Model

The motion unit of the robot can be divided in three parts, the controller, the motor, which includes the no linearity, 
and the mechanical part. The mechanical part (Fig. 6) of the mini robot has a behavior of a homonymic cube.

As demand values for the motion unit the velocity $v_{R}$ and angular velocity $\omega_{R}$ are given. To convert these values to the velocity of the left wheel $v_{R, L}$ and the right wheel $v_{R, R}$, which is used by the controller, the kinematic constraint for the wheels is used.

$$
\begin{aligned}
& v_{R}=\frac{v_{R, R}+v_{R, L}}{2} \\
& \omega_{R}=\frac{v_{R, R}-v_{R, L}}{B}
\end{aligned}
$$

$$
\begin{aligned}
& v_{R}=\dot{x} \\
& \omega_{R}=\dot{\varphi} \\
& v_{R, L} \\
& v_{R, R} \\
& B
\end{aligned}
$$

velocity of robot angular velocity of robot velocity of the left wheel velocity of the right wheel The gauge is the distance between the middle of the left and the middle of the right wheel

The encoder resolution of the DC motor is 512 impulses per rotation. This signal is a two-channel signal. The analysis of this signal quadruplicates the resolution. From this follows a signal of 2048 impulses per rotation.

$$
s_{\text {wheel }}=2 \cdot \pi \cdot i \cdot r_{\text {wheel }}
$$

$$
s_{\text {wheel }}=2 \cdot \pi \cdot \frac{12}{100} \cdot \frac{48}{2}=18.1 \mathrm{~mm} / \mathrm{rot}
$$

$$
\begin{array}{ll}
r_{\text {wheel }} & \text { Diameter of the wheel } \\
s_{\text {wheel }} & \text { The way the wheel covers per rotation } \\
& \text { of the motor } \\
i & \text { Gear ratio }
\end{array}
$$

The way the robot's wheel overs per rotation of the motor $s_{\text {wheel }}$ is $18.1 \mathrm{~mm}$. During this the encoder generates 2048 impulses. This means for the maximum velocity of the robot of $2.54 \mathrm{~mm} / \mathrm{ms}$ a resolution of 287 impulses. The number of impulses is a little bit higher than the number of different translatoric velocities per millisecond, which are 254.

Furthermore the sampling time has to be more less than $20 \mathrm{~ms}$, which is the time when the new demand value is set. The sampling time is set to $1 \mathrm{~ms}$.

For controlling the robot two independent controllers are implemented (Fig. 7), one per each motor and wheel respectively. The demand value, which was given as translatoric and angular velocity is converted to the velocity of the left and the velocity of the right wheel.

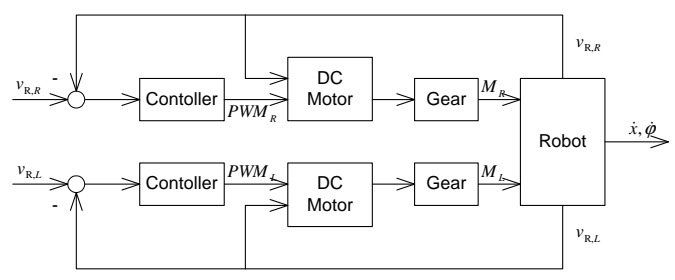

Fig. 7: Control Mechanism of the Robot

The controller of the mobile platform generates a PWM Signal $\left(P M W_{1}, P M W_{2}\right)$, which is equivalent to a direct current $u$. The motor converts this signal depending on its actual rotation speed $\left(\omega_{1}, \omega_{2}\right)$ into a torque $\left(\bar{M}_{1}, \bar{M}_{2}\right)$. The torque takes effect over a gear with a gear ratio $i$ on the wheel, which has the radius $R$. These torques $\left(M_{1}, M_{2}\right)$, which are effecting on the wheels, result to an acceleration $(\ddot{x}, \ddot{\varphi})$ of the robot. This leads to a wheel's velocity $\left(v_{R, L}, v_{R, R}\right)$ of the robot (Fig. 8).

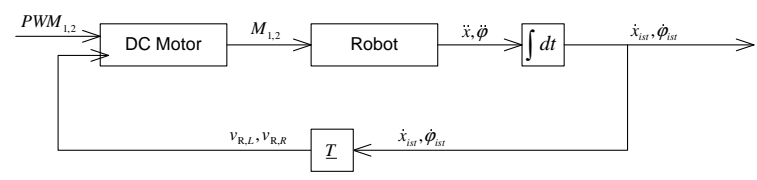

Fig. 8: DC motor

For controlling the robot a digital recursive PID controller (Fig. 9) is implemented. Other possible controllers were considered [5], however the recursive PID controller turned out as the best solution, if both motors are controlled separately.

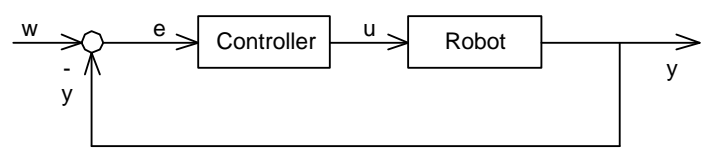

Fig. 9: Robot Controller

$$
\begin{array}{ll}
w & \text { demand value } \\
y & \text { feedback }
\end{array}
$$

$u(k)=u(k-1)+g_{0} \cdot e(k)+g_{1} \cdot e(k-1)+g_{2} \cdot e(k-2)$

$$
g_{0}=K_{R} \cdot\left(1+\frac{T_{D}}{T}\right)
$$




$$
\begin{aligned}
& g_{1}=-K_{R} \cdot\left(1+\frac{2 \cdot T_{D}}{T}-\frac{T}{T_{I}}\right) \\
& g_{2}=K_{R} \cdot \frac{T_{D}}{T}
\end{aligned}
$$

$\begin{array}{ll}u(k) & \begin{array}{l}\text { the manipulated variable for the step } \mathrm{k} \\ u(k-1)\end{array} \\ \begin{array}{l}\text { the manipulated variable for the step } \\ \text { before } \\ \text { the error for the step } \mathrm{k}\end{array} \\ K_{R} & \text { gain } \\ T_{D} & \text { derivative action time } \\ T_{I} & \text { integral action time } \\ T & \text { sampling time }\end{array}$

In order to calculate the next manipulated value it is necessary to store the last one. The control algorithm itself calculates only the difference of the last and the new manipulated value.

The actual velocities of the left $v_{L, \text { actual }}$ and the right wheel $v_{R, \text { actual }}$, the reference values of the controller, are calculated by the following equation:

$$
\begin{aligned}
& v_{L, \text { actual }}=\frac{n_{L, \text { actual }}}{x_{\text {Enkoder }}} \cdot \pi \cdot \frac{D_{R}}{2} \cdot i \\
& v_{R, \text { actual }}=\frac{n_{R, \text { actual }}}{x_{\text {Enkoder }}} \cdot \pi \cdot \frac{D_{R}}{2} \cdot i
\end{aligned}
$$

$$
\begin{array}{ll}
n_{L, \text { actual }} & \text { actual rotation speed in encoder im- } \\
& \text { pulses } \\
x_{\text {Enkoder }} & \text { Factor for the resolution of the encoder } \\
\frac{D_{R}}{2} \cdot i & \begin{array}{l}
\text { Diameter of the robots wheel multi- } \\
\text { plied with the gear ratio }
\end{array}
\end{array}
$$

\section{CONCLUSION AND FURTHER WORK}

Roby-Go was the first European MiroSOT soccer robot and became the prototype for nearly all European MiroSOT robots. In 2000 it became Vice European Champion. The robot was sold to the Czech Team of Brno and to the Slovenian Team of Ljubljana, too. The German team redesigned their not even finished robot completely based on Roby-Go. In 2001 the Czech copy became European Champion, Roby-Go itself took the third place. In the end of 2001 a revision of the electronic of Roby-Go was made. The robot was equipped with two additional 2-axis acceleration sensors ([4] and [2]) in order to prevent slipping wheels for faster acceleration. Speed wins in MiroSOT. In 2002 at the European Championship in the league "3 against 3 " as well as in the new formed league "5 against 5" the team from Brno succeeded and the team from Ljubljana became second. At the World Championship the new Roby-Go of the Austrian team took the 4th place. At the European Championship in 2003 in both categories the first three places were taken by Roby-Go and its derivates. At the World Championship 2003 a new league "7 against 7" was introduced. In this league Roby-Run took the second place and the Slovenian team became third. In the category 5 against 5 the robots took the second and fourth place. Roby-Run is the further development of IHRT (http://www.ihrt.tuwien.ac.at), the Austrian team. It has still the same electronic, but got a new mechanic. Roby-Go was sold to further teams, even to a team of the middle east.

Meanwhile the author has left the Austrian team and founded in summer of 2002 the Club for Promotion of Robot Soccer and Multi Agent Systems (http://www.robotsoccer.at). He was one of the initiators of CLARA (Consortium and Laboratory on Advanced Robotics Architectures), which was started in autumn 2003 at the Vienna University of Technology. Today six university institutes participate at CLARA. The robot is still being developed to a complete autonomous system. Step per step the whole functionality is shifted to the robot without enlarging its size. The robot exists now at version 6.0 codename Tinyphoon (The Worlds Most Powerful and Smallest Soccer Playing Robot with Integrated Digital Camera). The first version of Tinyphoon was introduced in autumn 2003 (http://www.tinyphoon.com).

\section{REFERENCES}

[1] G. Novak. Robot soccer: An example for autonomous mobile cooperating robots. In Proceedings of the First Workshop on Intelligent Solutions in Embedded Systems, pages 107-118, Vienna, Austria, June 2003.

[2] P. Putz. Antriebsregelung eines mobilen miniroboters. Master's thesis, Vienna University of Technology, Austria, May 2002.

[3] Official homepage http://www.fira.net of the organistor of the fira robot soccer tournaments.

[4] G. Novak. Multi Agent Systems - Robot Soccer. PhD thesis, Vienna University of Technology, Austria, April 2002.

[5] Novak G. Han, M.-W. Kopacek P. and Rojko A. Adaptive velocity control of mobile soccer robots. In Proceedings of the 9th International Workshop on ROBOTICS IN ALPEADRIA-DANUBE REGION - RAAD 2000, pages 41-46, Maribor, Slovenia, June 1-3 2000. 